\title{
Ion-beam modifications of the surface morphology and conductivity in some polymer thin films
}

\author{
M RAMAKRISHNA MURTHY* and E VENKATESHWAR RAO \\ Department of Physics, University College, Kakatiya University, Warangal 506 009, India
}

MS received 14 December 2001; revised 29 June 2002

\begin{abstract}
Studies on the surface micromorphology and surface conductivity in thin polymer films of poly vinyl alcohol (PVA) and poly ethylene oxide (PEO) in both as-grown and ion-implanted polymer films have been carried out to reveal certain specific features of the ordered state in these materials. Optical microscopic investigations revealed the existence and enhanced formation in number of spherulites and dendrites in ionimplanted films relative to the as-grown films. The number and rate of formation of spherulites indicated an increase in the degree of crystallinity in these films. Measurements of surface conductivity of as-grown and ion-implanted polymer films, employing four-point probe method, indicated a decrease in electrical conductivity on ion-implantation. Photomicrographic analysis of the PVA and PEO thin film surfaces, has enabled to propose a temperature-stress induced mechanism of crystallization in conjunction with the surface conductivity measurements. The decrease in surface conductivity on ion-implantation in both PVA and PEO thin films, is attributed to a decrease in mobility of macromolecular charged species due to an increase in degree of crystallinity as has been observed by optical microscopy.
\end{abstract}

Keywords. Ion-implantation; surface conductivity; polymer films; optical microscopy.

\section{Introduction}

Studies on ion-beam effects on polymers have gained significance in recent years, in view of the potential applications such as in surface science (Gerlach and Meyer 1981), phosphors (Jaworska et al 1989; Kaschny et al 1993) and integrated electronics (Skotheim et al 1998 ) etc. Drastic modifications of physical and surface properties have been reported (Loudiana et al 1986; Virk et al 2001), under different conditions on ion irradiation of polymers, a study of which reveal interesting phenomena with possible applications (Mazzoldi and Arnold 1987). The chemical constitution, stereoregularity, configuration and conformational aspects of polymers govern the specific surface features (Ward 1975), while the external conditions such as temperature, pressure or ionimplantation influence the physical states and the different physical properties (Grubb and Keller 1980; Young 1981; Geil 1983).

Experimental investigations on the ion beam modifications of surface conductivity in poly vinyl alcohol (PVA) and polyethylene oxide (PEO) are presented here. Studies on surface micromorphological modifications employing optical microscopy have also been made, the specific features of the ordered states attained on ion implantation is considered to effect the surface conductivity in thin films of these materials.

\footnotetext{
*Author for correspondence
}

\section{Experimental}

Commercially available poly vinyl alcohol $\left[\mathrm{CH}_{2} \mathrm{CH}(\mathrm{OH})\right]_{n}$ (molecular weight $\approx 14,000$ ) and poly ethylene oxide $\left[\mathrm{CH}_{2} \mathrm{CH}_{2} \mathrm{O}\right]_{n}$ (molecular weight $\approx 4000$ ), in the form of powder were employed in the present investigations.

Thin films of these materials were obtained by slow evaporation of very low concentrate solution in distilled water on glass slides. Growth and micromorphological features of these polymer surfaces were studied using a me-opta optical microscope.

$\mathrm{He}^{+}$ion-implantation has been carried out at the Materials Science Division, Indira Gandhi Centre for Atomic Research (IGCAR), Kalpakkam. A medium energy ion scattering (MEIS) accelerator with ion beam energy of $150 \mathrm{keV}$, a current of $500 \mu \mathrm{A}$ and dose rate of $10^{17}$ ions $/ \mathrm{cm}^{2}$ was employed. Ion-implantation in polymer films was carried out at room temperature under ultra high vacuum $\left(10^{-8} \mathrm{~m}\right.$ bar) for a few seconds.

Measurements of surface conductivity in both asgrown and ion-implanted polymer films were carried out, employing the four-point probe technique (Runyan 1975). This technique comprises a four-spring type, collinear and equally spaced probes, which are coated with zinc at the tips to ensure good electrical contact with the sample films. The probe spacing $(s)$ is $\sim 0.2 \mathrm{~cm}$, while the thickness $(t)$ of the films are $<0.076 \mathrm{~cm}$, thus the condition necessary for employing four-point probe method of measuring surface conductivity i.e. $t \ll s$, is satisfied. The surface on which the probes rest are mechanically 
lapped and these probes are mounted in a Teflon bush for good electrical insulation. Sample holder with four probes may be kept in an oven, to vary the temperature of the specimen. A thermocouple kept in contact with the specimen is used in conjunction with a digital temperature controller (DTC), to monitor the temperature of the sample to an accuracy of $\pm 1^{\circ} \mathrm{C}$. Any desired temperature can be set employing DTC and the samples were stabilized to these temperatures, prior to the conductivity measurements.

Different voltages $(V)$ are applied to the outer two probes and the resulting current $(I)$ is measured by connecting a Keithley electrometer amplifier (model $610 \mathrm{C}$ ) in series with inner two probes. Conductivity $(\sigma)$ was evaluated using the equation

$$
\sigma=I / 2 \pi s V \operatorname{mhos} \mathrm{cm}^{-1} \text {. }
$$
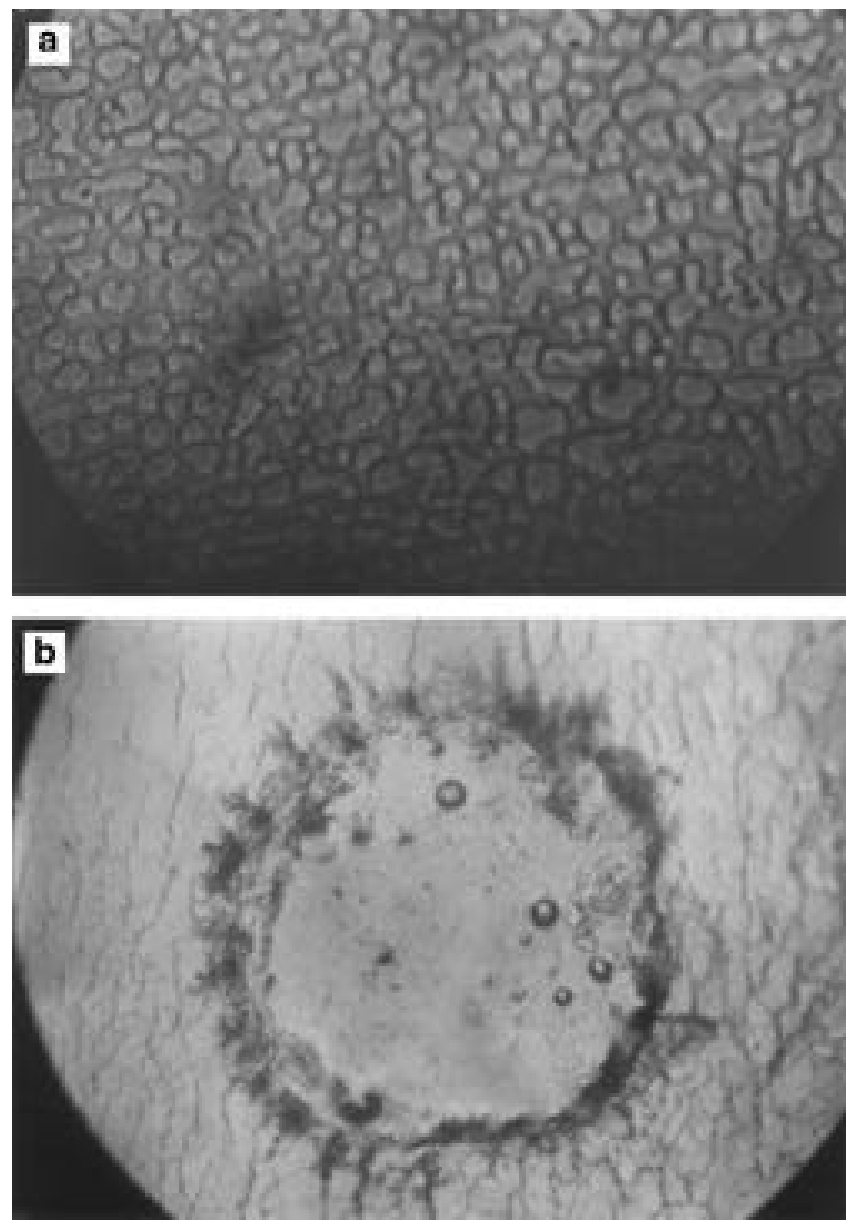

Figure 1. a. Optical micrograph of PVA thin film after $\mathrm{He}^{+}$ ion bombardment; observed under reflected light $(\times 200)$, the density of layered structures is very much less relative to the as-grown PVA film and b. optical micrograph of temperaturestress induced formation of spherulite in PVA thin film indicating the increased degree of crystallinity, in a selected region recorded under reflected light $(\times 450)$.

\section{Results and discussion}

Optical micrograph of the surface of PVA thin film on $\mathrm{He}^{+}$ion irradiation is as shown in figure 1a. After heating the film and applying a stress using a blunt circular indentor on the surface, the optical micrograph is as shown in figure $1 \mathrm{~b}$ from which it is inferred that there is an orderly alignment of macromolecules due to the formation of spherulitic structure by a temperature stress induced mechanism of crystallization.

Poly ethylene oxide (PEO) is a highly crystallizable polymer. The optical micrograph of an as-grown thin film surface of PEO is as shown in figure $2 \mathrm{a}$, revealed the formation of spherulites, indicating the presence of highly organized segregates of crystallites with a radial sector growth. The alignment of fibrils and symmetry in different sectors represent crystallinity of the PEO surfaces, as
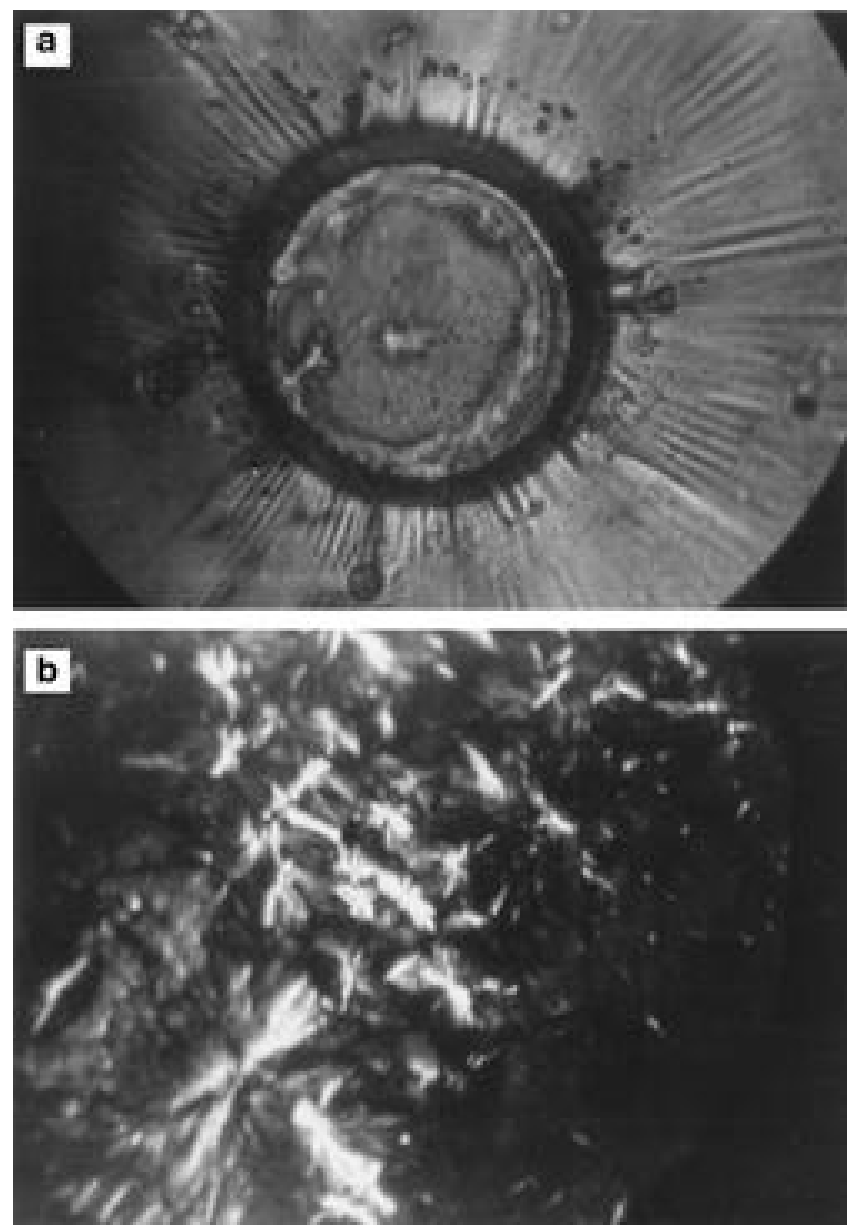

Figure 2. a. Optical micrograph of a spherulite in PEO thin film revealing the alignment of fibrils in an orderly way in different sectors. The radial sector growth of the spherulite is evident from the optical micrograph observed under reflected light $(\times 450)$ and b. optical micrograph of $\mathrm{He}^{+}$ion bombarded thin film of PEO as observed under crossed polarized light $(\times 450)$. 
has been observed earlier (Bassett and Keller 1962; Keith and Padden 1963; Bassett and Dammont 1964). The optical micrograph of $\mathrm{He}^{+}$ion bombarded PEO thin film is as shown in figure $2 b$; the formation of dendrites (tree like structures) indicated an increase in the degree of crystallinity.

Measurements of surface conductivity in both as grown and ion implanted PVA and PEO films have been carried out at different temperatures (from room temperature to near glass transition temperature), employing four-point probe method. Arrhenius plots (conductivity $\log \sigma$ in mhos $\mathrm{cm}^{-1}$ vs $1 / T, T$ in ${ }^{\circ} \mathrm{K}$ ) are as shown in figure 3 . Evaluation of activation energies from these plots showed an increase of $0.8 \mathrm{eV}$ and $1.1 \mathrm{eV}$, respectively for PVA and PEO on ion bombardment.

There has been a decrease in surface conductivity on ion bombardment, in both PVA and PEO films at all the observed temperatures. The decrease in electrical surface conductivity in polymers, in general, has been attributed to be due to an increase in the degree of crystallinity (Taylor and Lewis 1971; Finch 1973). The surface conductivity is found to decrease in PVA and PEO thin films on ion bombardment, which is further hastened by subsequent thermal stimulation.

Hence, it was thought that a relationship between the degree of crystallinity and conductivity may be established by an optical microscopic examination of surfaces of the samples, immediately after the measurements of electrical conductivity. Thus the ion bombarded films are subjected to a simultaneous thermal stimulation and stress as has been applied to the film by the array of probes. Such experimental conditions, however, did not allow the use of X-ray diffraction (XRD) technique to study the variation in crystallinity in these films.

The optical micrograph of as-grown PEO surface to which a stress has been applied employing a circular indentor at room temperature, is as shown in figure $4 \mathrm{a}$.

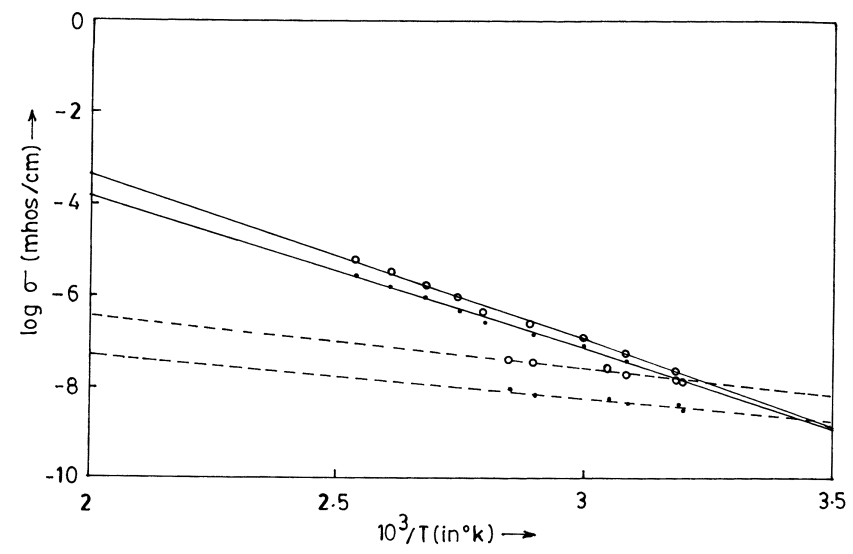

Figure 3. $\log (\sigma)\left[\mathrm{ohm}^{-1} \mathrm{~cm}^{-1}\right]$ conductivity vs $10^{3} / T$ plots for the as grown (o) and ion implanted (.) thin films of PVA (-) and PEO (----), depicting a decrease in conductivity on ion implantation as a function of temperature (measured in ${ }^{\circ} \mathrm{K}$ ).
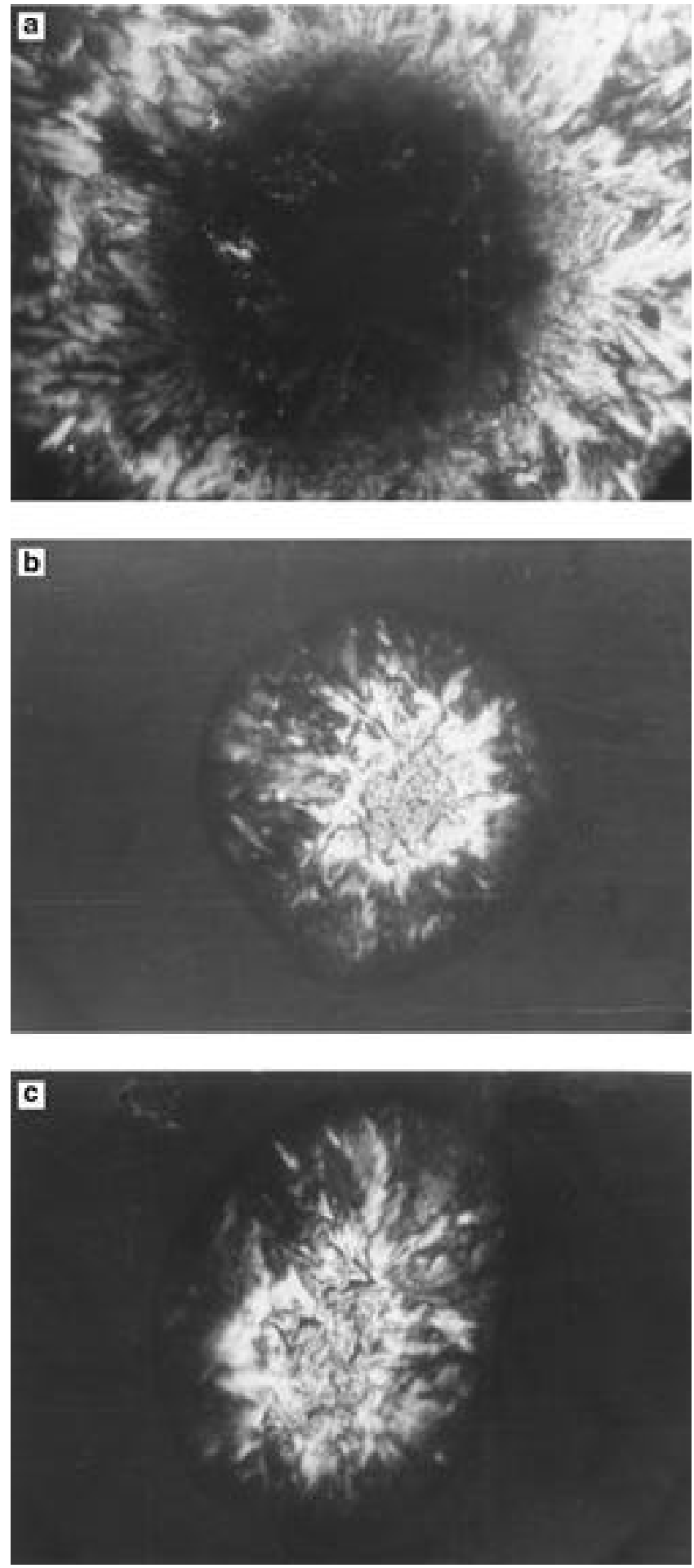

Figure 4. a. Optical micrograph of as grown surface of PEO thin film after indentation with a blunt circular indentor at room temperature observed under crossed polarized light $(\times 200)$. Formation of spherulitic region outside the indented region is due to stress induced crystallization mechanism, b and c. optical micrograph of irradiated PEO thin film recorded after measurement of surface conductivity at different temperatures observed under crossed polarized light $(\times 200)$. Formation of a large number of spherulites and dendrites within the circular indentor regions is due to temperature-stress induced crystallization. 
The central dark region represents the indentor mark, and the formation of spherulites outside the indentor mark have been observed due to stress induced crystallization. Figures $4 \mathrm{~b}$ and $4 \mathrm{c}$ show the formation of spherulites due to an increase in temperature followed by application of stress in ion-implanted PEO thin films. Spherulites were observed within the region of the indentors; incidentally, the indentors are the four probes used to measure surface conductivity. Thus there has been an increase in degree of crystallinity on ion bombardment, the process is hastened by a temperature-stress induced mechanism of crystallization.

Ion irradiation of polymers generate a number of charged species such as free radicals, ions and other low molecular weight charged species etc and the mobility of these species contribute to the surface conductivity.

The decrease in surface conductivity in both PVA and PEO thin films, on ion bombardment, is attributed to a decrease in the mobility of these molecular charged species due to an increase in the degree of crystallinity as has been revealed experimentally by optical microscopy.

\section{Acknowledgements}

The authors wish to thank IUC-DAEF, Indore, for financial support in the form of a research project and the Materials Science Division, IGCAR, Kalpakkam, for providing the accelerator facilities.

\section{References}

Bassett D C and Keller A 1962 Philos. Mag. 71553

Bassett D C, Dammont F R and Salovey R 1964 Polymer 5589

Finch C A 1973 Poly vinyl alcohol properties and application (Bristol: Arrow-Smith J W Ltd.) p. 340

Geil D H 1983 Polymer single crystals (New York: Interscience)

Gerlach U and Meyer O 1981 Surf. Sci. 103524

Grubb D T and Keller A 1980 J. Polym. Sci. Polym. Phys. Edn. 18207

Jaworska E, Wagner H and Diskorz Radiant W 1989 Phys. Chem. 33399

Kaschny J R, Amarlal L, Fink D and Behar M 1993 Radiation effects and defects in solids (USA: Gordon and Breach) 125 pp 289-298

Keith H D and Padden Jr. F J 1963 J. Appl. Phys. 342409

Loudiana M A, Soamid A and Dickinson J T 1986 Surf. Sci. 141409

Mazzoldi P and Arnold G W 1987 Ion-beam modification of insulators (Amsterdam: Elsevier) p. 301

Runyan W R 1975 Semiconductor measurements and instrumentation (Tokyo: Kogakusha Ltd., McGraw-Hill)

Skotheim T A, Elsenbaumer R L and Reyxold John R 1998 Handbook of conducting polymers (New York: Marcel Dekker) pp 589-638

Taylor D M and Lewis T J 1971 J. Phys. D (GB)4 1346

Virk H S, Chandi P S and Srivastava A K 2001 Bull. Mater. Sci. 24529

Ward I M 1975 Structure and properties of oriented polymers (London: Applied Science)

Young R J 1981 Introduction to polymers (London: Chapman and Hall) 